\title{
Nominalización deverbal y déficit informativo en los titulares periodísticos
}

\author{
Deverbal nominalization and informative \\ deficit in newspapers headlines
}

Juan NADAl Palazón

Universidad Nacional Autónoma de México

\begin{abstract}
RESUMEN: Entendida como el mecanismo de formación de sustantivos a partir de verbos, la nominalización deverbal es un rasgo característico del lenguaje periodístico, sobre todo en los titulares. La considerable frecuencia de uso de estas formas obedece a factores como la ideología subyacente y la economía lingüística. Particularmente destacable es la motivación ideológica, pues la elección de derivados nominales en lugar de oraciones completas revela, en muchos casos, la intención de omitir información relevante, como el agente o las circunstancias de la acción aludida.
\end{abstract}

ABSTRACT: Deverbal nominalization, understood as the mechanism to make nouns out of verbs, is a characteristic feature of the journalistic language, especially in headlines. The high incidence of these forms is due to factors such as the underlying ideology and linguistic economy. The ideological motivation particularly stands out, since the choice of nominal derivatives instead of complete sentences reveals, in many cases, the intention of omitting relevant information, such as the agent or the circumstances of the referred action.

Palabras Clave: titulares periodísticos, nominalización deverbal, déficit informativo, ideología, discurso.

KEY WORDS: newspaper headlines, deverbal nominalization, information deficit, ideology, discourse.

RECIBIDO: 27 de julio de 2014 • AcEPTADO: 4 de noviembre de 2014 

JuAn NADAl Palazón

Universidad Nacional Autónoma de México

\section{Nominalización deverbal y déficit informativo en los titulares periodísticos}

\section{Introducción}

Toda forma de comunicación verbal presupone una realización individual de la lengua, lo cual implica, entre otras decisiones, la elección de estructuras gramaticales. Dado el inventario de posibilidades de que disponen los hablantes para expresarse, la elección de determinada estructura gramatical, y no otra, es una cuestión significativa desde el punto de vista de la pragmática integrada y, desde luego, de la hermenéutica. ${ }^{1}$

Las elecciones de estructuras gramaticales responden a motivaciones ideológicas (Fowler 1986; Hodge y Kress 1993; Fairclough 1995, entre otros): como bien ha dicho Van Dijk (2003: 68-69), "las ideologías subyacentes [...] afectan las estructuras formales del lenguaje". En concordancia con ello, debe entenderse que, en toda forma de codificación lingüística, el enunciador orienta el sentido de su emisión

\footnotetext{
Diversos autores han abordado ya el tema de la coincidencia de objeto de estudio entre la hermenéutica y la pragmática. Dascal (1989) ha aportado buenos argumentos en esta dirección, y a él remito al lector interesado. Como bien observa Beuchot (2012: 52), la diferencia no radica ni en la finalidad ni en los conceptos de las categorías de análisis, sino en el origen (la pragmática procede de la filosofía analítica, y la hermenéutica, de la fenomenología). En cuanto a las coincidencias entre hermenéutica y análisis del discurso, véase el sugerente artículo de Bell 2011.
} 
hacia determinadas interpretaciones (Ducrot 1982 y 1980; Anscombre y Ducrot 1983). Ducrot afirma, desde esta perspectiva, que la lengua siempre da una aprehensión enunciativa del mundo, por cuanto la representación de la realidad pasa por el establecimiento previo de una serie de relaciones intersubjetivas dentro del discurso (Ducrot 1998: 124).

En cuanto actos de habla (cf. Austin 1962), las emisiones informativas poseen siempre un efecto perlocucionario. Si admitimos que la finalidad primordial del discurso periodístico es influir en el contenido y principios fundamentales de los conocimientos y representaciones sociales (cf. Romero 1996: 14), las configuraciones gramaticales características del discurso informativo deben responder a condicionamientos pragmáticos tendentes a imponer y reproducir posturas ideológicas (cf. Van Dijk 1990 y 2003).

En este trabajo analizo una de las peculiaridades lingüísticas distintivas del discurso periodístico: la transformación nominal (cf., entre otros, Steel 1971: 13; Casado Velarde 1978: 103; Van Dijk 1990: 117; Hurtado 2003: 110), que más adelante definiré como el mecanismo de formación de sustantivos a partir de vocablos pertenecientes a otras categorías gramaticales. Me baso en un corpus de 2397 titulares, publicados en los diarios mexicanos Reforma, El Universal, El Sol de México, La Jornada y La Prensa. ${ }^{2}$

Suele admitirse que, esquemáticamente, "los titulares son la parte más importante del texto" periodístico (Van Dijk 1997: 134), pues, además de sintetizar el texto que encabezan, orientan su interpretación (cf. Eco 1977: 165-187; Van Dijk 1990, 1997, 2006; Thogmartin 1991: 249-266). En muchos casos, sin embargo, su importancia es aún mayor: constituyen las únicas secuencias leídas por gran parte de los consumidores de diarios (Emig 1927; Gomis 1991; Martín Vivaldi 1998; López Hidalgo 2009). No en vano el titular es, para Steel (1971: 15), "la forma periodística por antonomasia" (cf. Alarcos 1977).

2 Dados los objetivos de investigación cualitativa de este artículo, no me ocupo de la descripción cuantitativa de los titulares con nominalizaciones deverbales. El análisis se centra, exclusivamente, en el estudio de usos observables en una amplia muestra de encabezados de nota informativa (Nadal 2009), género periodístico caracterizado, prototípicamente, por una jerarquización informativa de importancia descendente en el orden discursivo, y por la pretensión (nunca alcanzada totalmente, desde luego) de ocultar las huellas del sujeto enunciador. 
Me propongo evidenciar en las líneas siguientes que el uso de nominalizaciones deverbales en los titulares periodísticos encuentra a menudo su motivación en razones de índole pragmática, relacionadas sobre todo con la ideología del sujeto enunciador y con el principio general de economía lingüística. Mi exposición responde al orden siguiente: en primer lugar, defino el concepto de nominalización deverbal y proporciono una revisión muy general de algunos de los estudios que al respecto se han publicado; en segundo, refiero las características de la nominalización deverbal en los titulares periodísticos, así como los condicionamientos o motivaciones que mi análisis propone. Cierran unas conclusiones.

\section{Las nominalizaciones deverbales}

El término nominalización ${ }^{3}$ alude al proceso de formación de sustantivos o nombres a partir de una base perteneciente a otra categoría gramatical. Es, por tanto, un mecanismo de derivación. ${ }^{4}$ Esto ocurre, en español, gracias a la adición de sufijos a bases léxicas verbales o adjetivales. Así, por ejemplo, se obtiene el sustantivo recaudación al añadir el sufijo -(a)ción a la base del verbo recaudar, o el sustantivo simplismo, mediante la adición de -ismo a la base del adjetivo simple. Cuando la base a la que se adjunta el sufijo nominalizador es de naturaleza verbal, la nominalización se especifica como deverbal. En cambio, se califica como deadjetival cuando corresponde a un adjetivo la unidad que sirve de base para la derivación nominal. ${ }^{5}$

En esta ocasión profundizaré únicamente en ciertas nominalizaciones deverbales: las que suelen definirse como "acción y efecto"

"Formación sustantiva" para Alvar y Pottier 1983: 383.

4 Derivación: en términos generales, proceso morfológico de formación de léxico (Matthews 1980: 50) en el que hay adición, supresión o intercambio de sufijos ligados a una forma libre. Conlleva una alteración en el significado de la base y, en ocasiones, también un cambio de categoría gramatical (Varela Ortega 1992: 70). Los morfemas derivativos tienden a ser numerosos, pero suelen ocurrir en pocas combinaciones (Nida 1949: 99), a diferencia de los morfemas flexivos.

5 La voz nominalización también se ha hecho extensiva a la formación de nombres a partir de bases catalogadas, a su vez, como nominales: algunos autores llaman nominalizaciones denominales a derivaciones del tipo estoque > estocada. 
y que Comrie (1976: 178) llamó “nombres de acción” (action nominals). Las partículas -(a)ción, -sión, -ión, -miento, -aje y -ura son algunos de los morfemas nominalizadores de acción que tiene la lengua española. En el siguiente cuadro reúno varios ejemplos:

\begin{tabular}{|c|c|c|c|}
\hline Base verbal & $\begin{array}{c}\text { Sufijo } \\
\text { nominalizador }\end{array}$ & $\begin{array}{c}\text { Derivado } \\
\text { nominal }\end{array}$ & Significado \\
\hline grabar & -(a)ción & grabación & 'acción y efecto de grabar' \\
\hline aprehender & -sión & aprehensión & 'acción y efecto de aprehender' \\
\hline unir & -ión & unión & 'acción y efecto de unir' \\
\hline acercar & -miento & acercamiento & 'acción y efecto de acercar' \\
\hline camuflar & -aje & camuflaje & 'acción y efecto de camuflar' \\
\hline clausurar & -ura & clausura & 'acción y efecto de clausurar' \\
\hline maltratar & -0 & maltrato & 'acción y efecto de maltratar' \\
\hline
\end{tabular}

En los inicios de la gramática generativa, las nominalizaciones eran vistas como resultados de transformaciones (Chomsky 1957); posteriormente, Chomsky (1970: 184-221) propuso, en contra de esta idea, la "hipótesis lexicalista de la derivación de nominalizaciones de acción", según lacual éstas constituyen entradas separadas en el diccionario (basa su argumentación en las semejanzas sintácticas que en inglés existen entre estos derivados y los nombres no derivados). Años después, desde una perspectiva tipológica y luego de analizar datos en ruso, polaco, búlgaro, macedonio, árabe clásico, turco y uzbeko, Comrie (1976: 177201) determinó que lo importante es el grado de correspondencia que hay entre la estructura interna de una nominalización de acción y la de una frase nominal no derivada, atendiendo al modo como se presentan los argumentos, sujetos y objetos. ${ }^{6}$

Para Halliday (1994: 352), la nominalización es el "más poderoso recurso para crear metáforas gramaticales". ${ }^{7}$ En toda metáfora gramatical un componente semántico se construye en la gramática de manera no prototípica. Halliday propone dicho término para referirse

6 Koptjevskaja-Tamm (1993) ha adoptado la postura de Comrie y ha intentado establecer una tipología sintáctica de las nominalizaciones deverbales, con base en el análisis de un corpus con datos de setenta lenguas.

7 "Nominalizing is the single most powerful resource for creating grammatical metaphor" (Halliday 1994: 352). 
al "mismo significado" que se evoca por medio de otra clase de palabra, donde se produce una conjunción de dos niveles de significado como resultado de una nueva elección gramatical. Así, gracias a la nominalización de acciones, los procesos, normalmente expresados con verbos, se reformulan metafóricamente como sustantivos: según Koptjevskaja-Tamm (1993: 6), las formaciones sustantivas ocupan una posición intermedia entre los verbos y los sustantivos típicos, y comparten rasgos semánticos y discursivos con ambos.

En el mismo sentido, de acuerdo con Fowler (1986), la nominalización es un proceso organizativo del mensaje que reduce una oración a un nombre; de este modo, se aminora la transitividad y se pierde el conocimiento de los participantes personales. Por ejemplo, la palabra admisión esconde referencias del tipo "yo admito a usted", en enunciados como "la admisión de los aspirantes está sujeta a la disponibilidad de plazas", y muestra el proceso de admitir algo como estático, casi como si fuera un objeto.

Las nominalizaciones deverbales en los titulares periodísticos y algunos de sus condicionamientos

Bien conocida es la tendencia nominalizadora del discurso periodístico: Steel (1971: 13), por ejemplo, apunta con razón que, en él, "el grupo nominal adquiere una relevancia y una extensión mayor que en otros estilos de lengua" (cf. Van Dijk 1990: 117; Hurtado 2003: 110). Casado Velarde (1978: 103) destaca, por su parte, el reiterado uso de formaciones sustantivas en la confección de encabezados: "Con gran frecuencia en los titulares se presenta de forma nominalizada el hecho que se expresa con un verbo en el cuerpo de la noticia". Solamente como dato orientador, contiene derivados nominales 66\% (1589/2397) de los titulares que integran mi corpus.

La elevada incidencia de nominalizaciones de acción puede explicarse, en principio, por la indudable necesidad de ahorrar espacio o tiempo en los medios: cuando se usa el código metafórico, no solamente se está interesado en incorporar más información, sino en hacerlo de manera más económica. Esto es evidente en los encabe- 
zados, sobre todo si recordamos que su codificación lingüística es el resultado de un complejo proceso en el que incide, de manera definitiva, la tensión establecida por lo que Grice (1975) llamó la "máxima de cantidad", es decir, el principio que apunta a no expresar ni más ni menos información que la estrictamente requerida por los objetivos del intercambio en curso.

(1) a) Silencia Cuba el arribo del nuevo embajador soviético [JOR 12/12/ 91: 51]

b) Impugna Navarrete actuación del IEEM sobre presuntas facturas falsas del PAN [LAP 23/02/05: 8$]^{8}$

En muchos casos de nominalización de verbos intransitivos $-\mathrm{y}$ en algunos de transitivos - , todos los participantes aparecen expresados mediante modificaciones al núcleo sustantivo. Me refiero a casos como los de la serie (1), donde complementos adnominales refieren los agentes de las acciones expresadas por medio de nominalizaciones deverbales: en el ejemplo (1a), el nuevo embajador soviético es quien arriba, y en (1b), el Instituto Electoral del Estado de México es quien actúa. Se trata, desde luego, de casos de motivación puramente económica, como evidencia su cotejo con las secuencias de la serie (1'), integrada por construcciones hipotéticas:

(1') a) Arriba el nuevo embajador soviético a Cuba, y el gobierno de la Isla lo silencia

b) El IEEM actúa con respecto a presuntas facturas falsas del PAN, y Navarrete impugna

Las construcciones bajo ( 1 ') son, obviamente, más extensas que los enunciados de la serie (1). Los casos de (1') revelan que, en la

8 En todos los encabezados reproducidos en este trabajo he respetado fielmente la ortografía literal y puntual de los originales. He conservado también los usos de mayúsculas y minúsculas. Asimismo, he transcrito los encabezados en letras de espesor regular, bien entendido que los titulares siempre se imprimen en negrillas, cuyo uso se reserva aquí para destacar algunas palabras a las que se alude en el análisis. Entre corchetes añado la referencia del texto: coloco primero el nombre del periódico, abreviado y en mayúsculas; después de un espacio, la fecha, y por último, tras dos puntos, la página. Al final de este trabajo se encuentra la correspondiente lista de abreviaturas. 
enunciación de titulares de (1), se asume la existencia de ciertas oraciones cuyos contenidos se expresan, con un valor de contenido presupuesto, en grupos nominales que ejercen el oficio sintáctico de complemento directo. Secuencias como las de la serie (1') difícilmente se publicarían como titulares periodísticos, no solamente por su escasa economía lingüística, sino también porque las oraciones en posición focal - es decir, al principio - refieren, en estos casos, información consabida en el momento del acto de habla: se trata, claro está, de contenidos que aluden anafóricamente a otras ediciones del diario.

Las nominalizaciones de acción, sin embargo, suelen comportar la ausencia de información argumental. ${ }^{9}$ Esto puede deberse a dos razones: a que el periodista ignora la información o bien a que decide omitirla. Por ejemplo, en (2a) parece claro que el enunciador ignora quién es el agente de la acción nominalizada, y que considera irrelevante la identidad de las víctimas, acaso por conocida o genérica. En (2b), en cambio, parece que la generalización inherente al contenido noticioso seleccionado ocasiona la omisión de los numerosos agentes del maltrato infantil.

(2) a) Preocupa en León ola de asesinatos [sol 24/02/05: 8/B]

b) Niñas, víctimas del 95 por ciento de maltrato infantil [SOL 21/02/ 05: $10 / \mathrm{A}]$

En otros encabezados, la decisión de no mencionar información argumental parece responder a motivaciones de índole ideológica. En conformidad con el modelo del cuadrado ideológico (Van Dijk 2003), el acto de callar o expresar información conocida casi siempre encuentra su motivación en la ineludible existencia de una ideología subyacente; en el discurso de los medios de comunicación, ello supone, según este autor (2006), la imposición autoritaria de una visión del mundo.

Al igual que Van Dijk, Hodge y Kress (1993: 19 y ss.) consideran que es posible advertir algunos aspectos de la ideología del hablante si se atiende a la mención u omisión que éste hace de los participantes,

9 Procedente de la lógica de predicados, la denominación argumento se utiliza en gramática para referirse a cada uno de los sintagmas nominales que un predicado relaciona y que son requeridos por éste para efectuar una predicación completa. 
que normalmente están unidos por un proceso verbal: con las omisiones, concebidas por estos últimos autores como mecanismos de un modelo de producción textual llamado "no transaccional" (non transactive model), se producen enunciados comprensivos de una sola entidad del proceso aludido, la cual no siempre coincide con el agente o el experimentador. Se trata, según Fowler (1986), de procesos organizativos del mensaje que responden a pérdidas de interés informativo.

(3) a) Cobija operativo extorsión [REF 23/02/05: SB]

b) Por primera vez, pago íntegro a 18000 productores de frijol [LAP 21/02/05: 44]

c) En mayo, clausura definitiva de dos tiraderos en Nezahualcóyotl [SOL 25/02/05: 5/A,3.a]

d) Finaliza la identificación de víctimas del 11 de septiembre [JOR 24/02/05: 33]

En los casos (3a) a (3d), encontramos las voces extorsión, pago, clausura e identificación, respectivamente. En todos estos encabezados, la nominalización oculta información argumental muy importante desde el punto de vista informativo: ¿quién extorsiona a quién?, ¿quién paga cierta suma íntegra a 18 mil productores de frijol?, ¿quién clausura definitivamente dos tiraderos en Nezahualcóyotl?, ¿quién identifica a las víctimas del 11 de septiembre?

No parece que este tipo de déficit informativo responda a la intención de causar un extrañamiento tal en el alocutario que lo invite a consumir íntegramente el texto que el titular encabeza: a falta de información argumental, temporal, modal, aspectual y circunstancial, el proceso nominalizado se presenta la mayoría de las veces como si fuera espontáneo, a pesar de tratarse de derivados de formas léxicas cuya estructura semántica exige o presupone la existencia de entidades animadas que instigan, controlan y dirigen la acción representada.

El efecto de ambigüedad en la nominalización de acciones se debe a la habitual pérdida de argumentos y circunstancias, a la vez que de tiempo, aspecto, modo y modalidad verbales. El uso de esta metáfora gramatical apunta a un texto que, además de ambiguo, es estático: al convertir un proceso verbal en sustantivo, es decir, al mostrar una acción como si fuera un objeto, la relación entre procesos y partici- 
pantes se muestra inmóvil. Sin duda, estas propiedades son aprovechadas por los redactores de titulares para restar importancia a los agentes, que por lo general desempeñan una función sujetiva y, por tanto, cardinal en la "estructura profunda" del enunciado.

La omisión de algunos segmentos en el uso de estas entidades abstractas implica que el lector deba completar la información faltante en la fase de interpretación del titular, proceso que, como se sabe, no suele tomar en cuenta el texto que el encabezado rotula (cf. Emig 1927: 53-59; Eco 1977: 165-187; Casado Velarde 1984: 235-242; Hurtado 2003). Hodge y Kress (1993) consideran que la estructura profunda no siempre puede ser recobrada en su totalidad, por lo que los receptores frecuentemente interpretan los enunciados de maneras muy diversas: "El escritor presumiblemente conoce con exactitud el significado pleno, pero quizá el lector no", advierte Halliday (1994: 353). ${ }^{10}$

Analicemos más detalladamente el caso (3b). En este encabezado encontramos la forma pago, que constituye un derivado nominal del verbo pagar: ${ }^{11}$ sintácticamente, esta palabra constituye el núcleo del sujeto de una oración simple de predicado adverbial con verbo elíptico. Desde un punto de vista semántico, la "acción de pagar" recibe tres especificaciones en el contexto lingüístico: una temporalaspectual (A), que apunta a que nunca antes había ocurrido un suceso similar; una modal (в), que detalla la naturaleza integral del pago, y una alusiva al supuesto paciente de la acción referida (C), es decir, los 18 mil productores de frijol. $\frac{\text { Por primera vez, pago íntegro }}{\text { A }} \frac{18000 \text { productores de frijol }}{\text { B }}$

A: especificación temporal-aspectual

B: especificación modal

C: especificación de paciente

Como hemos dicho, el titular no especifica el agente del proceso: no sabemos quién pagó, por primera vez, una cierta cantidad íntegra a 18 mil productores de frijol. Una lectura a la nota informativa

10 "The writer presumably knows exactly what it means; but the reader may not".

11 Productores es también una nominalización, pero de momento no nos interesa. 
permite saber que se trata de la Secretaría de Agricultura, la cual, además, resulta ser el origen de la información procesada. Desde una perspectiva pragmático-hermenéutica, esto parece revelar la intención de restar importancia al agente de una acción cuya referencia presenta, en principio, connotaciones positivas. Bien sugiere Van Dijk (2003) que el omitir o disimular información relacionada con logros ajenos supone rivalidades o posturas discordantes con relación a la entidad responsable.

Esta interpretación encuentra sustento en el hecho de que se enfatiza la especificación temporal-aspectual del derivado nominal, esto es, el contenido expuesto que denota la naturaleza inaugural de la acción. Situada en posición focal, es decir, al principio del encabezado, esta información supone que las autoridades agrarias no han cumplido, históricamente, con algo que el contexto presenta como una obligación gubernamental. Apoya esta lectura la especificación modal, de la cual se deriva la conclusión de que los pagos anteriores han sido incompletos. Todo esto presenta a los 18 mil campesinos, más que como beneficiarios de la acción nominalizada, como víctimas de abuso de las autoridades.

\section{Conclusiones}

Primera. La complejidad de los titulares periodísticos es, con frecuencia, el resultado de una alta densidad léxica y de una fuerte nominalización, en la que los procesos verbales están metafóricamente codificados como estructuras nominales. Tal situación se explica por el hecho de que la nominalización permite reducir oraciones completas a sustantivos que se incorporan de manera normal a cualquier estructura sintáctica. La alta incidencia de las nominalizaciones deverbales en los titulares periodísticos responde a factores concurrentes en la enunciación, tales como la ideología subyacente, la adaptabilidad del lenguaje y el principio de economía lingüística.

Segunda. La nominalización de acciones suele producir un efecto de ambigüedad en el enunciado. Esto se debe a que normalmente hay pérdidas de información argumental, circunstancial, temporal, 
modal y aspectual; la omisión del agente suele dar por resultado que el proceso referido parezca espontáneo. Además, el convertir un proceso verbal en sustantivo ocasiona que la relación entre procesos y participantes se muestre inmóvil.

Tercera. La elección de derivados nominales en lugar de oraciones completas parece revelar, en muchos casos, la existencia de una estrategia persuasiva que comporta la omisión de datos relevantes, como el agente o las circunstancias de la acción referida. Por cuanto el uso de estas formas suele ser una consecuencia de la actitud que el periodista adopta ante los contenidos informativos durante el proceso de construcción de la noticia, el análisis de este tipo de fenómenos parece develar posturas ideológicas y, por ende, líneas editoriales.

Cuarta. Los periodistas interpretan la realidad, la fragmentan y la presentan en períodos y en unidades normalmente completas e independientes (Gomis 1991: 35-47). A su vez, los titulares son interpretaciones condensadas de esos relatos, pues, en términos de Van Dijk (1990), por lo general expresan la macroestructura semántica del relato contiguo. Dado que los titulares periodísticos son las únicas secuencias leídas por la mayoría de los consumidores de diarios, el uso de nominalizaciones deverbales en ellos, con la correspondiente pérdida de información argumental, constituye un factor que conduce a interpretaciones parciales de los procesos noticiosos por parte de los lectores, con evidentes consecuencias en la formación del presente social de referencia de la acción colectiva.

\section{Bibliografía}

Alarcos Llorach, Emilio (1977). "Lenguaje de los titulares", en Lenguaje en periodismo escrito, F. Lázaro Carreter (ed). Madrid, Fundación Juan March (Serie Universitaria, 37).

Alvar, Manuel y Bernard PotTIER (1983). Morfología histórica del español. Madrid, Gredos (Biblioteca Románica Hispánica, III: Manuales, 57).

Anscombre, Jean-Claude y Oswald Ducrot (1983). L'argumentation dans la langue. Bruxelles, Pierre Mardaga.

Austin, John Langshaw (1962). How to do things with words. Cambridge, Harvard University Press. 
BeLl, Allan (2011). "Re-constructing Babel: Discourse analysis, hermeneutics and the interpretative arc", Discourse Studies, 13 (5).

Beuchot, Mauricio (2012). "Hacia una pragmática analógica", Acta Poetica, 33 (1).

Casado Velarde, Manuel (1978). "La transformación nominal, un rasgo de estilo de la lengua periodística", Cuadernos de Investigación Filológica, 4 (1).

- (1984). "Semiótica de los titulares: pautas para el análisis de los titulares periodísticos", en Teoría semiótica. Actas del Congreso Internacional sobre Semiótica e Hispanismo, vol. I, M. A. Garrido Gallardo (ed.). Madrid: Csic.

Chomsky, Noam (1957). Syntactic structures. The Hague, Mouton.

- (1970). "Remarks on nominalization", en R. Jacobs y P. Rosenbaum (eds.). Readings in English transformational grammar. Waltham, Ginn.

Comrie, Bernard (1976). "The syntax of action nominals: a cross-language study", Lingua, 40.

DASCAL, Marcelo (1989). "Hermeneutic interpretation and pragmatic interpretation", Philosophy and Rhetoric, 22 (4).

Ducrot, Oswald [1972] (1982). Decir y no decir. Trads. Walter Minetto y Amparo Hurtado. Barcelona, Anagrama (Biblioteca de Lingüística, 2).

- (1980). Les échelles argumentatives. Paris, Minuit.

- [1995] (1998). "Componentes de la descripción lingüística", en O. Ducrot y J. M Shaeffer (eds.), Nuevo diccionario enciclopédico de las ciencias del lenguaje. Trads. María Camino Girón, Teresa María Rodríguez y Marta Tordesillas. Madrid, Arrecife.

Eco, Umberto (1977). "Guida all'ipretazione del linguagio giomalistico", en L. Renzi y M. A. Cortelazzo (eds.), La lingua italiana oggi: un problema scolastico e sociale. Bologna, Socie Editrice il Mulino (Problemi e prospective. Serie di linguistica e critica litteraria).

EMIG, Elmer (1927). "The connotation of newspaper headlines", Journalism Quarterly, 4 (4).

FAIRCLOUgh, Norman (1995). Media discourse. London, Edward Arnold.

FOWLER, Roger (1986). Linguistic criticism. Oxford, Oxford University Press.

Gomis, Lorenzo (1991). Teoría del periodismo: cómo se forma el presente. México: Paidós Mexicana (Paidós Comunicación, 44).

GrICE, Herbert Paul (1975). "Logic and conversation", en P. Cole y J. L. Morgan (eds.), Syntax and Semantics 3: Speech Acts. New York, Academic Press.

Halliday, Michael Alexander Kirkwood (1994). An introduction to functional grammar. 2. ${ }^{\text {a }}$ ed. London, Edward Arnold.

HodGe, Robert y Gunther KRess (1993). Language as ideology. 2. ed. New York, Routledge.

Hurtado GonzÁlez, Silvia (2003). El uso del lenguaje en la prensa escrita. Valladolid, Universidad de Valladolid (Lingüística y Filología, 56). 
KoptJevskaja-TAmm, Maria (1993). Nominalizations. London-New York, Routledge (Theoretical Linguistics).

López Hidalgo, Antonio (2009). El titular. México, Alfaomega.

MalchuKov, Andrej L. (2004). Nominalization / verbalization: constraining a typology of transcategorial operations. Munchen, Lincom (Studies in Language Typology, 8).

Martín Vivaldi, Gonzalo (1998). Géneros periodísticos. 6. ed. Madrid, Paraninfo.

Matthews, Peter H. (1980). Morfología. Introducción a la teoría de la estructura de la palabra. Trad. Rafael Monroy Casas. Madrid, Paraninfo.

NADAl Palazón, Juan (2009). El discurso ajeno en los titulares de la prensa mexicana. México, Universidad Nacional Autónoma de México.

NidA, Eugene A. (1949). Morphology. The descriptive analysis of words. 2. ${ }^{a}$ ed. Ann Arbor, The University of Michigan Press.

Romero Álvarez, María de Lourdes (1996). "El relato periodístico como acto de habla", Revista Mexicana de Ciencias Políticas y Sociales, 169.

SteEl, Brian (1971). "Los estilos funcionales y la enseñanza del idioma", Español Actual, 18: 9-18.

THOGMARTIN, Clyde (1991). "The pragmatics of french newspaper headlines", en J. Verschueren (ed.), Levels of linguistic adaptation. Amsterdam-Philadelphia, John Benjamins (Pragmatics \& Beyond, New Series, 6: 2).

VAN DIJK, Teun Adrianus [1980] (1990). La noticia como discurso. Trad. Guillermo Gal. Barcelona, Paidós (Paidós Comunicación, 41).

- [1988] (1997). “Cómo se lleva una minoría a los titulares? Minorías étnicas en la prensa", en Racismo y análisis crítico de los medios. Trad. Montserrat Bast Kraan. Barcelona, Paidós (Paidós Comunicación, 82).

- (2003). Ideología y discurso. Barcelona, Ariel (Ariel Lingüística).

- (2006). "Discurso y manipulación: discusión teórica y algunas aplicaciones", Signos, 60: 49-74.

Varela Ortega, Soledad (1992) Fundamentos de morfología. Madrid, Síntesis (Lingüística, 5).

Abreviaturas

$\mathrm{JOR}=$ La Jornada

LAP $=$ La Prensa

$\mathrm{REF}=$ Reforma

$\mathrm{SOL}=$ El Sol de México

$\mathrm{UNI}=$ El Universal 
